\title{
Protocolos de Investigação de Variáveis Psicológicas na Epilepsia Infantil ${ }^{1}$
}

\author{
Paula Teixeira Fernandes ${ }^{2}$ \\ Elisabete Abib Pedroso de Souza \\ Universidade Estadual de Campinas
}

\begin{abstract}
RESUMO - A epilepsia é uma condição crônica comum na infância, cujo diagnóstico mostra dificuldades psicossociais e de ajustamento familiar, que parecem estar relacionadas com crenças e qualidade da interação pais-filhos. Este trabalho teve como objetivo esquematizar estratégias de investigação para as variáveis familiares e psicológicas: crenças, impacto da doença, relacionamento familiar, identificação de mudanças. A partir do levantamento de relatos dos pais de crianças com epilepsia e de aspectos da literatura, foram elaborados questionários psicológicos para identificar as importantes variáveis que afetam a vida da criança com epilepsia e sua família. Diante deste contexto, pode-se concluir que o uso de protocolos de investigação mais adequados facilita a avaliação psicológica e garante a coleta de dados.
\end{abstract}

Palavras-chave: epilepsia infantil; protocolos; relacionamento familiar; avaliação psicológica.

\section{Investigation Protocols of Psychological Variables in Infantile Epilepsy}

\begin{abstract}
Epilepsy is a common chronic condition in the childhood and its diagnosis reveals psychological, social and family difficulties, that seem to be related with beliefs and quality of parents-children interaction. The purpose of this paper is to schematize investigation strategies for the psychological variables: beliefs, impact of the disease, family relationship, identification of changes. Based upon collected reports of epileptic children's parents and upon surveyed aspects of the literature, psychological questionnaires were elaborated to identify important variables that affect the child's epilepsy life and his family. The use of more appropriate investigation procedures facilitates the psychological evaluation and ensures the collection of data.
\end{abstract}

Key words: infantile epilepsy; investigation protocols; family relationship; psychological evaluation.

A epilepsia infantil é uma das doenças crônicas mais comuns na infância e seu diagnóstico mostra dificuldades psicossociais, comportamentais e de ajustamento familiar, que parecem estar relacionadas a crenças irracionais e à qualidade da interação pais e filhos.

As crenças irracionais são idéias errôneas baseadas em preconceitos, não possuem base científica e acabam trazendo sentimentos e atitudes negativas para o paciente e sua família. Os medos e as expectativas dos pais parecem contingentes a estigmas ligados à epilepsia. As características da doença afetam diretamente a relação pais e filhos, aumentando os comportamentos de ansiedade e superproteção dos pais (Ford, Gibson \& Dreifuss, 1984; Lothman \& Pianta, 1994; Souza \& Guerreiro, 1996). Consequentemente, a dinâmica familiar traz uma alta prevalência de comportamentos alterados, dificuldades de aprendizagem, alterando o ajustamento psicossocial da criança e sua qualidade de vida (Austin, 1989; Seidenberg, Beck \& Geisser, 1986), que é um conceito subjetivo da pessoa sobre aquilo que ela tem e pode ser definido como senso de bem-estar físico, psicológico e social (Silva \& De Marchi, 1997; Souza \& Guerreiro, 1996).

1 Trabalho realizado com apoio da FAPESP

2 Endereço: Rua Antonio Lapa n¹125, CEP: 13025-242, Campinas S.P. E-mail: paulatfb@terra.com.br
Para se fazer uma avaliação adequada de pessoas com epilepsia, é preciso considerar não apenas os aspectos relacionados à doença (tipo, frequência e severidade das crises, etiologia, etc), mas também os aspectos comportamentais, psicológicos, culturais, sociais, emocionais e a qualidade de vida.

A partir de trabalhos realizados (Souza, Nista, Scotoni \& Guerreiro, 1998) e de relatos dos pais de crianças com epilepsia, verificou-se a necessidade de se criar instrumentos em que todas estas áreas pudessem ser investigadas, para haver um tratamento mais efetivo com prevenção de comportamentos inadequados e consequente redução do stress e melhora na qualidade de vida, promovendo assim um melhor ajustamento das crianças e suas famílias.

Este trabalho teve como objetivo esquematizar estratégias de investigação específicas para as variáveis psicológicas, relativas a crenças, ao impacto da doença na família, ao relacionamento familiar, a identificação de mudanças.

\section{Os Protocolos}

A partir do levantamento de relatos dos pais de crianças com epilepsia (em grupos de apoio realizados no Ambulatório de Epilepsia Infantil da FCM/Unicamp, desde 1997) e de temas da literatura (Souza \& cols., 1998; Fernandes \& 
Souza, 1999), foram elaborados protocolos psicológicos de investigação para identificar aspectos importantes que afetam a vida da criança portadora de epilepsia e sua família: as variáveis psicológicas e familiares e o impacto da doença na dinâmica familiar e no comportamento da criança.

Os protocolos de investigação estão divididos em:

- Pré-teste: protocolo de identificação de crenças; escala de impacto da doença na família; protocolo de investigação do relacionamento criança-família.

- Pós-teste: protocolo de investigação de mudanças.

\section{Protocolo de identificação de crenças}

Este protocolo tem como objetivo a identificação de idéias e preconceitos ligados à epilepsia e ao comportamento da criança.

São exemplos de questões deste protocolo, que pedem respostas do tipo sim/não: "A epilepsia é uma doença contagiosa?; Você acha que as crises ocorrem porque seu filho tem algum verme?; Tem medo que seu filho fique com alguma doença mental?; Você acha que ele pode melhorar com passes ou benzimentos?"

A epilepsia, como outras condições crônicas, é caracterizada pela incerteza e pelo seu significado social. Muitas pessoas não possuem um conhecimento profundo a respeito desta desordem e algumas idéias não possuem base científica. Embora alguns saibam que as crises refletem algo anormal na atividade do cérebro, mitos e preconceitos sobre causas e consequências ainda existem (Devinsky \& Penry, 1993; Baker, Jacoby, Buck, Stagis \& Monnet, 1997; Gumnit, 1997). Esta falta de informação a respeito da epilepsia e seu tratamento mobiliza as pessoas a desenvolverem respostas negativas em relação à doença, o que gera idéias e crenças irracionais, tais como: a epilepsia causa retardo mental, a epilepsia é contagiosa, é causada por masturbação excessiva ou por algum tipo de verme, é espiritual etc. Estas idéias, originadas de diversas fontes, trazem pensamentos negativos que, por sua vez, trazem comportamentos inapropriados, influenciando negativamente o ajustamento psicossocial das pessoas.

\section{Escala de impacto da doença na família}

Esta escala visa a identificação de sentimentos e reações que a epilepsia causa na família, os fatores mais estressantes e as áreas mais afetadas (social, familiar, escolar, saúde, autoconceito).

As questões desta escala referem-se à etapa mais difícil, sentimentos diante das crises, etc. Em uma das questões, por exemplo, pergunta-se: "Qual foi a etapa mais difícil?" Para responder, são oferecidas as seguintes alternativas: "quando o filho teve a primeira crise, quando soube que o filho tinha epilepsia, durante o tratamento, outros". Também há espaço para comentários.

Em uma outra questão, é perguntado: "O que você sentiu quando seu filho teve a primeira crise? Por que sentiu isso?". Como alternativas de respostas, são oferecidas: "medo, raiva, tristeza, confusão, nervoso, desapontamento, mágoa, ansiedade, vergonha, negação, susto, rejeição, culpa".

A epilepsia pode causar um impacto no ajustamento social da criança e sua família e, como consequência, uma pobre adaptação da criança ao tratamento da epilepsia. Pesquisas realizadas com pais indicam que a epilepsia impõe um peso não só para a criança, mas também para a família, restringindo atividades e aumentado responsabilidades para os pais e irmãos (Hoare \& Kerley, 1991). As primeiras respostas de stress à epilepsia - medo, raiva, confusão, tristeza - são perfeitamente entendidas e normais, por isso é importante que sejam identificadas e discutidas, para que se consiga diminuir o impacto negativo desta desordem na vida dos pacientes e suas famílias.

\section{Protocolo de investigação do relacionamento criança e família}

Neste protocolo, busca-se identificar os comportamentos e atitudes dos filhos e dos pais, antes e depois do início das crises, as relações interpessoais da criança (com os irmãos, com os pais, com os amigos).

Alguns exemplos de perguntas deste protocolo referemse a: "Você acha que o comportamento do seu filho era diferente antes dele começar a ter crises?; Como é o relacionamento dele com os irmãos?; Você percebe alguma relação entre a sua atitude e o comportamento do seu filho?".

A qualidade da interação entre pais e filhos produz consequências no bem-estar da criança, no seu desempenho escolar, nas suas relações sociais, nas suas atividades. Estudos mostram que as famílias que possuem crianças portadoras de epilepsia ficam com baixos níveis de auto-estima, comunicação e suporte familiar e altos níveis de stress e ansiedade (Austin, 1988; Lothman \& Pianta, 1993). A família que possui um filho portador de epilepsia tem menos proximidade e mais restrições de comportamentos, atividades e comunicação. As crianças portadoras de epilepsia são tratadas com superproteção e dependência; os pais as tratam como doentes e acreditam que qualquer atividade que as deixem nervosas pode precipitar uma crise (Thompson \& Upton, 1994). Outros estudos, como o de Keith (1978), também evidenciaram nos pais sentimentos de inadequação, raiva, susto, culpa, confusão e atitudes de superproteção. Muitas vezes, o excesso de cuidados é tanto que os pais acabam se esquecendo dos outros membros da família.

\section{Protocolo de investigação de mudanças}

Neste protocolo busca-se a identificação de alterações nos pais a nível de comportamentos, atitudes interacionais e crenças, em relação a informações anteriores a respeito da epilepsia.

Um exemplo de questão deste protocolo é: "Estas informações modificaram algumas crenças que você possuía sobre a epilepsia?". A resposta pede sim/não e quais as crenças que realmente mudaram. 
Quando se identifica precocemente as variáveis psicológicas relacionadas à epilepsia, é possível atuar de maneira mais efetiva e positiva na dinâmica familiar, controlando o ajustamento da criança e sua família (Fernandes \& Souza, 1999). É importante identificar se houve mudança na percepção dos pais em relação a seus próprios comportamentos e atitudes, aos comportamentos dos filhos e à própria doença. Estudos (Souza \& cols., 1998) mostram que os pais sentem-se mais capacitados a lidar com seus filhos após serem informados sobre a doença.

$\mathrm{O}$ uso de procedimentos de investigação mais adequados facilita a avaliação psicológica e garante a coleta de dados. Tais instrumentos necessitam serem testados para avaliar a consistência do conteúdo.

\section{Referências}

Austin, J.K.(1988). Childhood epilepsy: Child adaptation and family resources. Journal of Child and Adolescent Psychiatry Mental Health Nursing, 1, 18-24.

Austin, J.K. (1989). Comparison of child adaptation to epilepsy and asthma. Journal of Child and Adolescent Psychiatry Mental Health Nursing, 2, 139-144.

Baker, G.A.; Jacoby, A.; Buck, D.; Stalgis, C \& Monnet, D. (1997). Quality of Life of people with epilepsy: A european study. Epilepsia, 38, 353-362.

Devinsky, O. \& Penry, J.K. (1993). Quality of life in epilepsy: The clinician's view. Epilepsia, 34 (suppl 4), S4-S7.

Fernandes, P.T. \& Souza, E.A.P. (1999). Inventário Simplificado de Qualidade de Vida na Epilepsia Infantil: primeiros resultados. Arquivos de Neuropsiquiatria, 57(1), 40-43.

Ford, C.A., Gibson, P. \& Dreifuss, F.E. (1984). Psychosocial considerations in childhood epilepsy. In Dreifuss, F.E. (Org.), $P e-$ diatric epileptology classification and management of seizures in the child, (18, pp. 277-295). Massachussets: John Wright \& Sons Ltda.

Gumnit, R.J. (1997). Living well with epilepsy, New York: Demos Vermande.

Hoare, P. \& Kerley, S. (1991) Psychosocial adjustment of children with chronic epilepsy and their families. Developmental Medicine Child Neurology, 33, 201-215.

Keith, R.M. (1978). The feelings and behavior of parents of handicapped children. Developmental Medicine Child Neurology, 4, 524-527.

Lothman, D.J. \& Pianta, R.C. (1993). Role of child-mother interaction in predicting competence of children with epilepsy. Epilepsia, 34, 658-669.

Lothman, D.J. \& Pianta, R.C. (1994). Predicting behavior problems in children with epilepsy: Child factors, disease factors, family stress and chid-mother interaction. Child development, $65,1415-1428$

Seidenberg, M., Beck, N. \& Geisser, M. (1986). Academic achievement of children with epilepsy. Epilepsia, 27, 753-759.

Silva, M.A.D. \& De Marchi, R. (1997). Qualidade de vida e promoção da saúde. Em Saúde e Qualidade de Vida no trabalho, (2, pp. 25-30). São Paulo: Editora Best Seller.

Souza, E.A.P. \& Guerreiro M.M. (1996). Qualidade de vida e epilepsia. Em Epilepsia, Guerreiro C.A.M. e Guerreiro M.M. (Org.), São Paulo: Lemos editorial, 18, 191-199.

Souza, E.A.P., Nista, C.R., Scotoni, A.E. \& Guerreiro, M.M. (1998). Sentimentos e reações de pais de crianças epilépticas. Arquivos de Neuropsiquiatria, 56(1), 39-44.

Thompson, P.J. \& Upton D. (1994). Quality of life in family members of persons with epilepsy. In: Trimble M.R., Dodson W.E. (Org.) Epilepsy and quality of life, (2, pp. 19-31). New York: Raven Press. 